\title{
Application of novel targeting nanoparticles contrast agent combined with contrast-enhanced computed tomography during screening for early-phase gastric carcinoma
}

\author{
KAIMIN ZHANG ${ }^{1}$, XIJIAN DU ${ }^{2}$, KAIHU YU $^{2}$, KAIYU ZHANG $^{3}$ and YICHENG ZHOU ${ }^{4}$ \\ ${ }^{1}$ Physical Examination Center; ${ }^{2}$ Department of Radiology, Xianning Central Hospital; \\ ${ }^{3}$ Department of Radiology, The First People's Hospital of Xianning City, Xianning, Hubei 437000; ${ }^{4}$ Department of \\ Radiology, Tongji Medical College, Huazhong University of Science Tongji Hospital, Wuhan, Hubei 430030, P.R. China
}

Received July 23, 2016; Accepted May 5, 2017

DOI: $10.3892 /$ etm.2017.5388

\begin{abstract}
Gastric cancer is one of the most common human tumors worldwide. The biggest bottleneck is a lack of advanced and sensitive protocols for the diagnosis of patients with early-stage gastric cancer. Therefore, more sensitive methods of diagnosing gastric cancer are urgently required to improve survival rates. In this clinical study, contrast-enhanced computed tomography (CECT) with targeting nanoparticles contrast agent (CECT-TNCA) was used to diagnose early-stage gastric cancer. The specific-targeted tyrosine kinase inhibitors of gastric cancer, including platelet-derived growth factor receptor- $\beta$, Ret and Kit, were used as TNCAs. A total of 484 patients with suspected gastric cancer were voluntarily recruited to investigate the efficacy of CECT-TNCA in the diagnosis of patients with early-stage gastric cancer. Patients with suspected gastric cancer were subjected CT and CECT-TNCA to detect whether gastric tumors existed. TNCA was orally administered before CT and CECT-TNCA (20 min). Our diagnostic data revealed that CECT-TNCA improved sensitivity and provided a new protocol to diagnose tumors in patients with suspected gastric cancer at the early stage. In addition, imaging using CECT-TNCA enabled the visualization of tiny nodules in the gastric area. CECT-TNCA diagnosed 182 patients with suspected gastric cancer as tumor-free. CECT-TNCA confirmed gastric cancer in 302 patients. Our novel diagnosis indicated significantly $(\mathrm{P}<0.01)$ differential signal enhancement in the gastric nodules via CECT-TNCA compared with $\mathrm{CT}$, suggesting higher accuracy and the accumulation of TNCA in tumor nodules in the
\end{abstract}

Correspondence to: Professor Xijian Du, Department of Radiology, Xianning Central Hospital, 228 Jingui Road, Xianning, Hubei 437000, P.R. China

E-mail: duxijiansci@163.com

Key words: gastric cancer, early diagnosis, nanoscale microbubble, contrast-enhanced computed tomography-targeting nanoparticles contrast agent, specific-targeted tyrosine kinase inhibitors $\beta$ stomach. Furthermore, survival rates of patients detected by early-diagnosis of CECT-TNCA were significantly higher than the mean five-year survival $(\mathrm{P}<0.01)$. In conclusion, our investigations demonstrate that the sensibility and accuracy of CT is improved through combination with liposome-encapsulated nanoparticle contrast agent for the diagnosis of early stage gastric cancer when compared with single CT detection. CECT-TNCA improves the accuracy of CT and diagnostic confidence in assessing mural enhancement in patients with suspected gastric cancer.

\section{Introduction}

Gastric cancer is one of the most common human epithelial malignancies and remains the second leading cause of cancer-associated mortality for clinical cancer (1). Clinical statistics and investigations have shown that $>70 \%$ of new cases of gastric cancer-associated deaths occur in developing countries (2,3). Gastric cancer exhibits higher morbidity and mortality rates than other carcinoma derived from the digestive system as gastric cancer is more invasive $(4,5)$. A previous study has indicated that the five-year survival rate for gastric cancer is $<80 \%$ (6).

Currently, the majority of clinical patients with gastric cancer are categorized as having an advanced stage once diagnosed. In addition, although reports have shown that apoptosis-resistance of gastric cancer is inevitable in the development of cancer progression, the apoptotic resistance of gastric cancer cells in patients with gastric cancer has been indicated as the most important hurdle to overcome in clinical treatment $(7,8)$. Furthermore, apoptotic resistance has become the greatest challenge in cancer therapy due to fierce resistance of tumor cells though various types of molecules mechanism (9-11). Although several comprehensive therapies exist that induce apoptosis, which is the most important component of treatment for patients with gastric cancer, the survival rate remains low (12). These previous reports focused on the efficacy of targeted molecular therapies, and the early diagnosis of gastric cancer is often overlooked.

In recent years, contrast-enhanced ultrasound, computed tomography, fl tomography, ont-positron emission, and 
tomography (FDG-PET) has been widely used in the diagnosis of human cancer (13). Although many advantages of contrast-enhanced ultrasound have been presented, its relatively reduced resolution compared with $\mathrm{CT}$ means that it cannot confirm the final diagnosis for patients with suspected gastric cancer (14). However, nanoscale microbubbles have been used to improve the resolution of ultrasound, as they resonate when exposed to ultrasound waves $(14,15)$. In addition, computerized tomography and chip technology are the most common methods used to diagnose patients with suspected cancer (16). However, the efficacy of single computerized tomography is limited to diagnosing patients with early phase tumors. Furthermore, contrast agent combined with CT for the analysis of tumor biology has been studied and achieved adequate efficacy (17). Therefore, we hypothesized that specific-targeted nanoscale microbubbles may contribute to the efficacy and resolution of CT in the diagnosis of patients with suspected gastric cancer.

In the present study, CECT combined with targeting nanoscale microbubble contrast agent (CECT-TNCA) was introduced to detect the early stage of patients with suspected gastric cancer. Notably, CECT in conjunction with ultrasound contrast further expanded its application in the field of primary diagnosis and confirmed diagnosis (18). This clinical analysis demonstrated the potential application of CECT-TNCA for imaging modality and sensitivity improvements in the diagnosis of gastric cancer. Our outcomes indicated many advantages of CECT-TNCA in both early diagnosis and final confirmation of suspected cases when compared to single CT detection.

\section{Materials and methods}

Ethics statement. The design of this clinical study was carried out in strict accordance with the approval and recommendations in the Guide for the Care and Use of clinical study of Xianning Central Hospital (XNCH: 20091108A4). All surgery and euthanasia protocols were standardized. All patients provided written informed consent.

Patients. A total of 484 patients with suspected gastric cancer aged 14.8-65.2 years were recruited for this prospective analysis, in which the follow-up period was 60 months. The number of male (222) and female (264) patients was approximately equal. Furthermore, 236 healthy subjects (male, 124; female, 112) aged 24.0-62.6 years were recruited. Biochemical parameters of patients with suspected NSCLC and healthy subjects recruited between May 2012 and June 2015 were eligible for further analysis. All patients were subjected to scanning for the detection of early-stage gastric cancer by CECT and CECT-TNCA. All healthy subjects had no cancer history or gastric diseases. Patients with cancer history were excluded in the present study.

Nanoparticles contrast agent. A novel liposome-encapsulated nanoparticles contrast agent containing multiple targets was introduced for diagnosing patients with early-stage gastric cancer. Platelet-derived growth factor receptor- $\beta$ (PDGFR- $\beta$ ), Ret, and Kit bound with the nanoparticles of superparamagnetic iron oxide particles via covalent bonds described in a
Table I. Characteristics of patients with suspected gastric cancer.

\begin{tabular}{lcc}
\hline Characteristics & Male & Female \\
\hline Patients (n) & 222 & 264 \\
Age (years) & $14.8-65.2$ & $21.6-62.2$ \\
Medical history of cancer (n) & 3 & 5 \\
Blood pressure (mmHg) & $110.2 \pm 12.8$ & $113.4 \pm 10.3$ \\
Blood glucose (mmol/l) & $7.7 \pm 3.6$ & $8.2 \pm 3.2$ \\
Diagnosis (n) & & \\
CECT-TNCA & 222 & 264 \\
CECT & 222 & 264 \\
\hline
\end{tabular}

CECT-TNCA, contrast-enhanced computed tomography-targeting nanoparticles contrast agent.

Table II. Confirmation of the dosage of targeting nanoparticles contrast agent required for diagnosis.

\begin{tabular}{lccc}
\hline Variable & $\begin{array}{c}1-10 \mathrm{mg} / \mathrm{kg} \\
(\mathrm{n}=16)\end{array}$ & $\begin{array}{c}11-20 \mathrm{mg} / \mathrm{kg} \\
(\mathrm{n}=24)\end{array}$ & $\begin{array}{c}21-30 \mathrm{mg} / \mathrm{kg} \\
(\mathrm{n}=20)\end{array}$ \\
\hline $\begin{array}{l}\text { Signal intensity } \\
\text { (HU) }\end{array}$ & $76.5 \pm 7.2$ & $92.5 \pm 5.8$ & $93.4 \pm 6.4$ \\
Sensitive (\%) & $64.4 \pm 17.3$ & $86.3 \pm 10.4$ & $83.8 \pm 9.5$ \\
\hline
\end{tabular}

previous study (19). Nanoparticles contrast agent and Optison (GE Healthcare, Chicago, IL, USA) were orally administered to ensure that they covered each corner of the stomach prior to CECT and CECT-TNCA (30 min). Following administration with CECT-TNCA, the TNCA was distributed in the stomach. Microbubbles contained targeting nanoparticles contrast agent with the capacity to target travelling tumor cells, which acted as an accurate tracer for tumor cells (20). TNCA was located in the lesion following administration with CECT-TNCA. After $30 \mathrm{~min}$, the TNCA was visualized via CECT. No side effects were observed in patients exposed to TNCA.

Scan protocol. A CECT diagnosis system was used to analyze CECT clinical trials using preprogrammed settings. Preprogrammed settings were optimized to achieve optimal image formation. CECT was performed on the stomachs of all patients according to manufacturers instructions (Philips Medical Systems, Inc., Bothell, WA, USA). Details of the principles and settings of contrast-enhanced ultrasound were described in a previous study (21). In addition, CECT-TNCA imaging was performed in all patients with suspected gastric cancer.

Data analysis. Data from CECT-TNCA image sets was analyzed using the ADMIRE CECT system (version 3.10; Siemens Healthineers, Erlangen, Germany). Volume of tumors was measured by CECT-TNCA imaging. All patients with suspected early stage gastric cancer were analyzed by CECT-TNCA and CT. Gastric tumor nodules were 
A

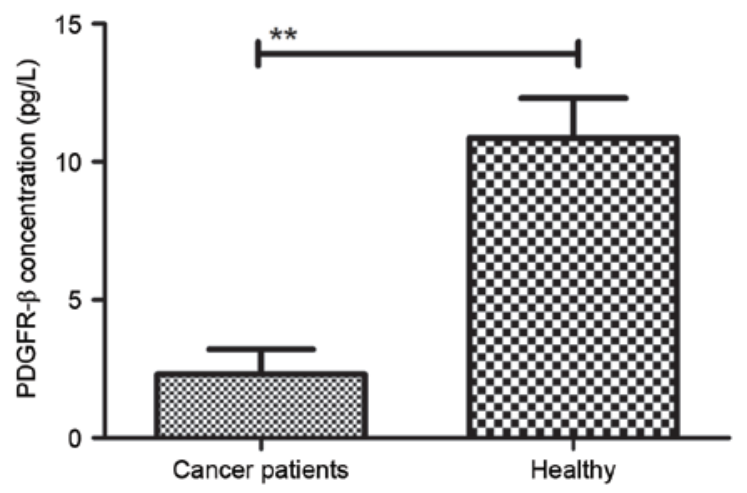

B

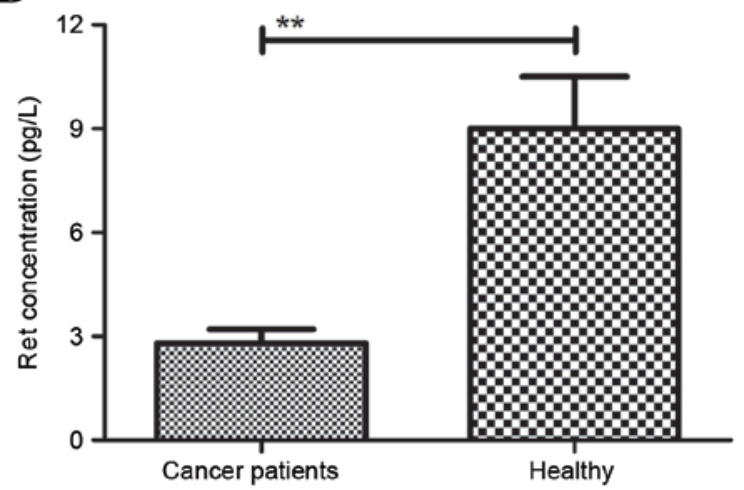

C

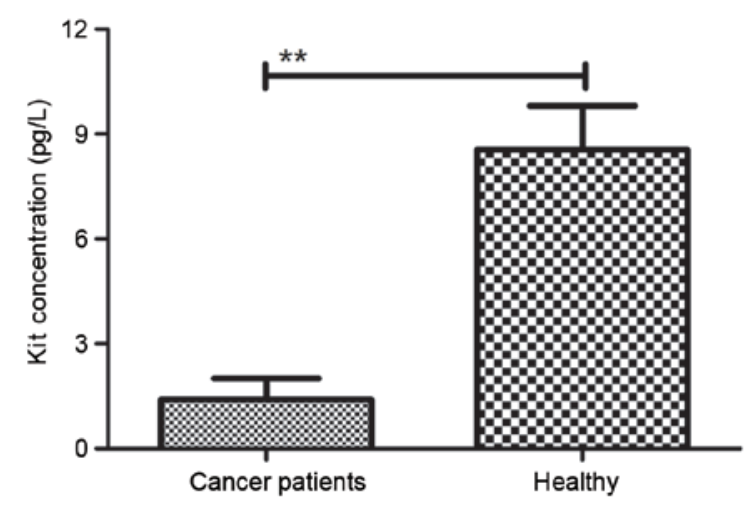

Figure 1. Analysis of plasma concentrations of (A) PDGFR- $\beta$, (B) Ret and (C) Kit between patients with gastric cancer and healthy subjects. ** P $<0.01$ vs. control (Student's t-test). PDGFR- $\beta$, platelet-derived growth factor receptor- $\beta$.

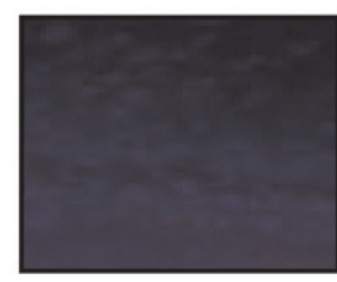

Control

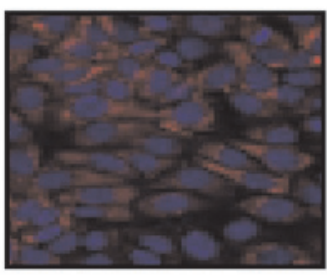

RET

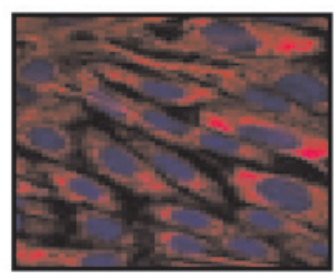

PDGFR- $\beta$

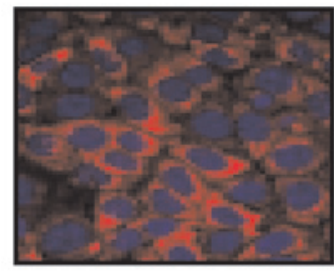

KIT
Figure 2. Immunostaining assay of PDGFR- $\beta$, Ret and Kit for gastric cancer cells from patients with gastric cancer. PDGFR- $\beta$, platelet-derived growth factor receptor $\beta$ (magnification, $\mathrm{x} 40$ ).

observed and tumor size was automatically calculated using Sante CT Viewer (version 2.0; Santesoft, Ltd., Athens, Greece).

Treatment of patients with gastric cancer diagnosed by CECT-TNCA. Patients with early stage gastric cancer diagnosed by CECT-TNCA received various different treatments

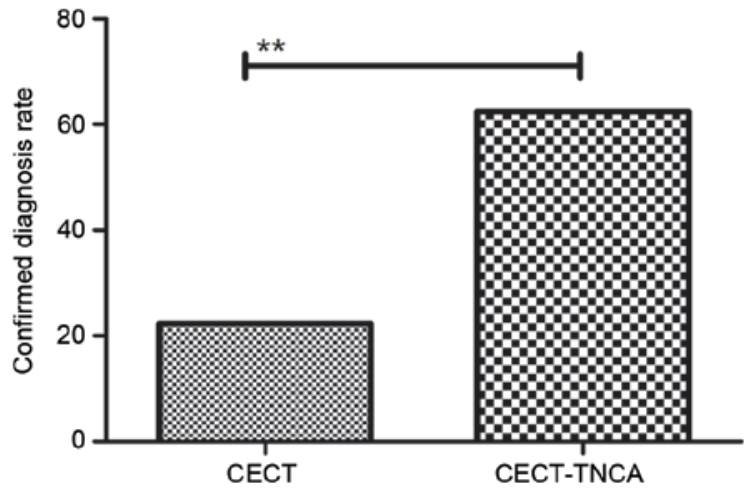

Figure 3. Comparison of the confirmed diagnosis rate determined by CECT and CECT-TNCA. ${ }^{* *} \mathrm{P}<0.01$ vs. control (Student's t-test). CECT-TNCA, contrast-enhanced computed tomography-targeting nanoparticles contrast agent.

including radiotherapy $(\mathrm{n}=39)$, chemotherapy $(\mathrm{n}=49)$, Chinese medicine $(n=58)$, biological therapy $(n=35)$, and comprehensive therapy $(\mathrm{n}=53)$. Median overall survival and median progression-free survival were analyzed as previously described (22).

Immunofluorescence and histological staining. Following diagnostic confirmation via CECT-TNCA, tumor cells from patients with gastric cancer were cultured in vitro with Dulbecco's modified Eagle's medium supplemented with 10\% heat-inactivated FBS (Invitrogen; Thermo Fisher 
A

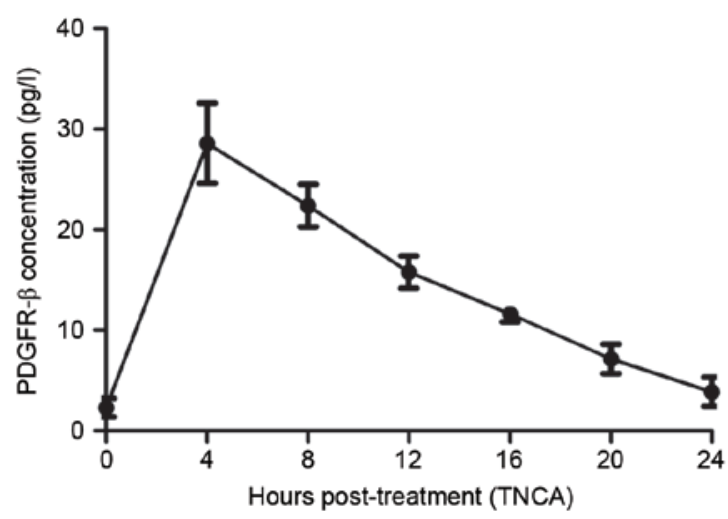

B

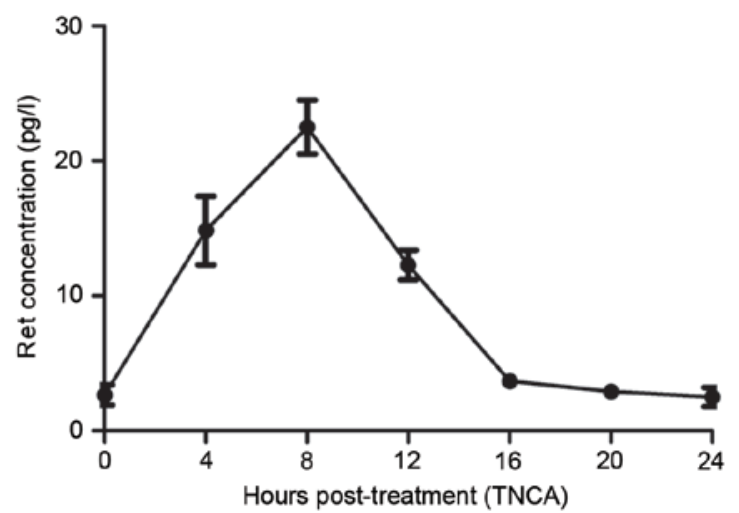

C

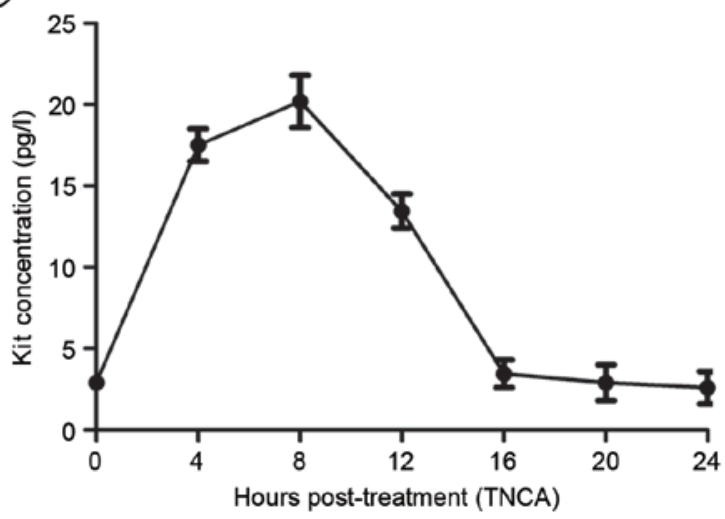

Figure 4. Metabolism of (A) PDGFR- $\beta$, (B) Ret and (C) Kit in targeting nanoparticles contrast agent in serum in patients who underwent CECT-TNCA. CECT-TNCA, contrast-enhanced computed tomography-targeting nanoparticles contrast agent.

Scientific, Inc., Waltham, MA, USA). Gastric tumors cells $\left(1 \times 10^{6}\right.$ cells $\left./ \mathrm{ml}\right)$ were incubated with TNCA for $30 \mathrm{~min}$ at $37^{\circ} \mathrm{C}$. Cells were observed under a fluorescence microscope. Immunofluorescence procedures were previously reported in detail (23). For histological staining, tumor sections were stained with hematoxylin and eosin staining as previously reported (24).

Statistical analysis. All data were presented as the mean and standard deviation of triplicate experiments. Unpaired data was determined by Student's t-test and comparisons of data between multiple groups were analyzed by variance. Kaplan-Meier was used to estimate the survival rate during 60 -month long-term follow-up observations. $\mathrm{P}<0.05$ was considered to indicate a statistically significant difference.

\section{Results}

Plasma concentrations of PDGFR- $\beta$, Ret, and Kit in patients with suspected gastric cancer. In order to analyze the target characteristic of TNCA, a total of 484 patients with suspected gastric cancer were voluntarily recruited to investigate the efficacy of CECT-TNCA in the diagnosis of patients with early-stage gastric cancer. Characteristics of patients with suspected gastric cancer are summarized in Table I. The dose of TNCA to achieve the optimum efficiency was identified as $15 \mathrm{mg} / \mathrm{kg}$ (Table II). In addition, we investigated the plasma concentration of PDGFR- $\beta$, Ret, and Kit in patients with suspected gastric cancer. As shown in Fig. 1A, PDGFR- $\beta$ plasma concentration was significantly lower in patients with gastric cancer when compared with healthy subjects. We also found that plasma concentration of Ret was significantly downregulated in patients with gastric cancer when compared with healthy subjects (Fig. 1B). In addition, compared with healthy subjects, plasma concentration of Kit was significantly lower in patients with gastric cancer (Fig. 1C). Furthermore, clinical analysis also indicated that our novel TNCA containing PDGFR- $\beta$, Ret, and Kit adhered to gastric cancer cells, which contributed to the signal strength and resolution (Fig. 2).

Efficacy of CECT-TNCA for the early diagnosis of patients with suspected gastric cancer. As shown in Fig. 3, clinical analysis showed that 182 patients $(37.6 \%)$ were diagnosed as tumor-free and 302 patients (62.4\%) were identified as having gastric cancer, as determined by CECT-TNCA. However, the positive rate of patients with gastric cancer was only $22.3 \%$ (108 patients) when evaluated via CECT alone. In addition, the investigation found that TNCA increased the plasma concentration of PDGFR- $\beta$ and metabolized within $24 \mathrm{~h}$ (Fig. 4A). Patients who underwent CECT-TNCA exhibited increasing plasma concentrations of Ret that peaked at $12 \mathrm{~h}$ and attenuated by $16 \mathrm{~h}$ (Fig. 4B). Plasma concentrations of Kit were increased and metabolized within $20 \mathrm{~h}$ (Fig. 4C). These clinical data indicated that CECT-TNCA is an efficient 


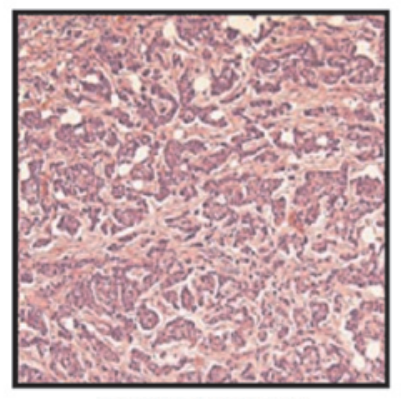

Cardiac carcinoma

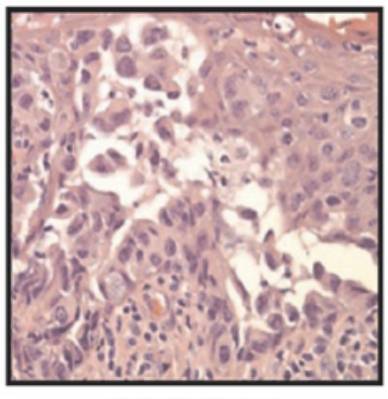

Gastric cancer

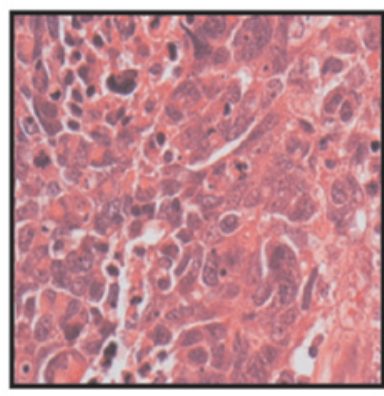

Pyloric carcinoma

Figure 5. Histological staining analyses the types of gastric cancers in patients diagnosed by CECT-TNCA. CECT-TNCA, contrast-enhanced computed tomography-targeting nanoparticles contrast agent (magnification, $\mathrm{x} 40$ ).

diagnostic strategy for the early diagnosis of patients with suspected gastric cancer.

Histopathology analysis of the accuracy of CECT-TNCA-diagnosis patients with gastric cancer. After diagnosing patients with suspected gastric cancer, histopathology analysis was used to further confirm diagnosis. Representative cardiac carcinoma, gastric cancer and pylorus carcinoma incidence rates were studied in the patients who had a confirmed diagnosis of gastric cancer. As shown in Fig. 5, our data showed that histopathology analysis identified cardiac carcinoma, gastric cancer and pyloric carcinoma gastric cancer. The incidence rate of cardiac carcinoma, gastric cancer and pyloric carcinoma was $33.8 \%$ (102 cases), $47.0 \%$ (142 cases) and $19.2 \%$ (58 cases), respectively, in gastric cancer (Fig. 6). These outcomes suggest that the CECT-TNCA method is accurate and sensitive for diagnosing patients with gastric cancer.

Survival rate of patients with gastric cancer diagnosed by CECT-TNCA. Patients with early-phase gastric cancer received different treatments to inhibit tumor cell growth or eradicate gastric cancer. We analyzed the reports of the treatment methods and survival rates of patients with gastric cancer diagnosed by CECT-TNCA. Characteristics of 234 patients with early-phase gastric cancer diagnosed by CECT-TNCA are summarized in Table III. At the 60-month follow-up, we observed that 132 patients $(56.4 \%)$ were tumor-free and 88 patients $(37.6 \%)$ had survived and exhibited tumors. The mortality rate was $6.0 \%$ (14 patients; Table IV). Median overall survival was 45.8 months (Fig. 7; range, 30.4-63.8 months) and median progression-free survival was 36.8 months (Fig. 8; range, 24.5-52.4 months). These data indicate that patients with early-phase gastric cancer received anti-cancer treatments that prolonged the survival and progression-free survival period, and that that comprehensive therapy had notable therapeutic effects compared with the others treatments.

\section{Discussion}

Cancer early diagnosis is the biggest obstacle in human cancer treatment $(25,26)$. In recent years, contrast-enhanced ultrasound, fluorodeoxyglucose-positron emission, tomography (FDG-PET), CECT and chip technology have been widely used in the diagnosis of human cancer $(27,28)$. In particular, CECT and chip technology present more advantages than

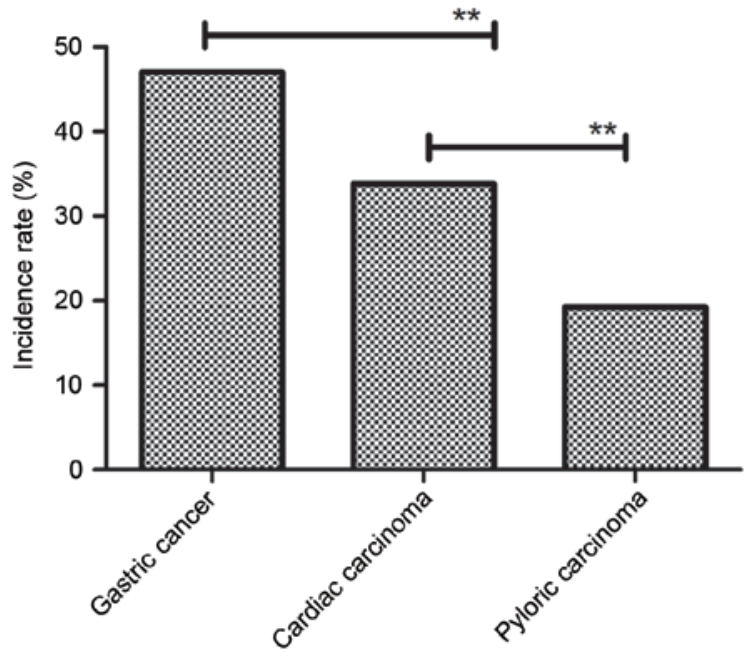

Figure 6. Analysis the incidence rate of the types of gastric cancers in patients diagnosed by CECT-TNCA. One-way analysis of variance revealed a significant effect. ${ }^{* *} \mathrm{P}<0.01$ vs. control. CECT-TNCA, contrast-enhanced computed tomography-targeting nanoparticles contrast agent.

other diagnostic methods $(29,30)$. However, the application of gene chip technology is restricted due to expensive detection and the professional analysts required $(31,32)$. Therefore, CECT has been become the most prevalent diagnostic method in the majority of hospitals worldwide $(33,34)$.

Though CECT has been widely applied in the diagnosis of human cancer, the accuracy and sensitivity of CECT is insufficient for the detection of early-stage tumors $(35,36)$. Barium sulfate and iodinated contrast media are frequently used for angiography studies and the diagnosis of tumors in the digestive system $(37,38)$. In addition, many electropositive iron and iron oxide nanoparticles are used as contrast media and have been reported to be useful in the diagnosis of human cancer in previous clinical trials $(39,40)$. Furthermore, retroreflective-type Janus microspheres have also been reported as a novel contrast agent for enhanced optical coherence tomography (41). However, these contrast mediums only improve the partial accuracy of CT in a certain degree. Therefore, elucidating more efficient contrast mediums with targeting characteristics has attracted increasing attention from researchers and clinicians in the field of cancer research and clinical therapy.

In the present study, we introduced a comprehensive approach of CECT combined with target nanoparticles 
Table III. Treatment of patients with gastric cancer diagnosed by CECT-TNCA.

\begin{tabular}{lcc}
\hline Characteristics & Male & Female \\
\hline Patients (n) & 114 & 120 \\
Age (years) & $15.6-56.2$ & $22.4-52.8$ \\
Medical history of cancer (n) & 2 & 2 \\
Blood pressure (mm Hg) & $108.6 \pm 8.5$ & $107.5 \pm 9.8$ \\
Blood glucose (mmol/l) & $8.4 \pm 1.5$ & $7.6 \pm 2.4$ \\
Treatments (n) & & \\
Radiotherapy & 18 & 21 \\
Chemotherapy & 25 & 24 \\
Chinese medicine & 32 & 26 \\
Biological therapy & 18 & 17 \\
Comprehensive therapy & 21 & 32 \\
\hline
\end{tabular}

CECT-TNCA, contrast-enhanced computed tomography-targeting nanoparticles contrast agent.

Table IV. Survival of patients diagnosed by CECT-TNCA after 60-month follow-up.

\begin{tabular}{lccc}
\hline Treatment & Tumor-free & $\begin{array}{c}\text { Survived with } \\
\text { tumor(s) }\end{array}$ & DNS \\
\hline Radiotherapy & 12 & 22 & 5 \\
Chemotherapy & 25 & 20 & 4 \\
Chinese medicine & 37 & 18 & 3 \\
Biological therapy & 19 & 15 & 1 \\
Comprehensive therapy & 39 & 13 & 1 \\
\hline
\end{tabular}

CECT-TNCA, contrast-enhanced computed tomography-targeting nanoparticles contrast agent; DNS, did not survive.

contrast agent to improve the accuracy for patients with suspected gastric cancer in the early stage. Target nanoparticles contrast mediums, containing PDGFR- $\beta$, Ret, and Kit, were encapsulated by liposome. Our clinical outcomes indicate that liposome-encapsulated TNCA presents a potential tumor-specific approach that may lead to improvements in the diagnostic accuracy of patients with early-stage gastric cancer. Targeted binding of liposome-encapsulated TNCA with gastric tumor cells enhances signal intensity in the lesions of the stomach, resulting in an improvement in the spatial resolution of CECT. Notably, pharmacokinetic tracer kinetics analysis demonstrated that the target nanoparticles contrast mediums of PDGFR- $\beta$, Ret, and Kit were metabolized within $24 \mathrm{~h}$. No side effects were noted during the diagnostic period. Long-term follow-up reports showed that patients diagnosed by CECT-TNCA at the early stage present higher median overall survival (30.4-63.8 months) and median progression-free survival (24.5-52.4 months).

Tyrosine kinase inhibitors of PDGFR- $\beta$, Ret, and Kit-mediated angiogenesis have been identified as key factors in the development of human cancer $(42,43)$. PDGFR- $\beta$, Ret,

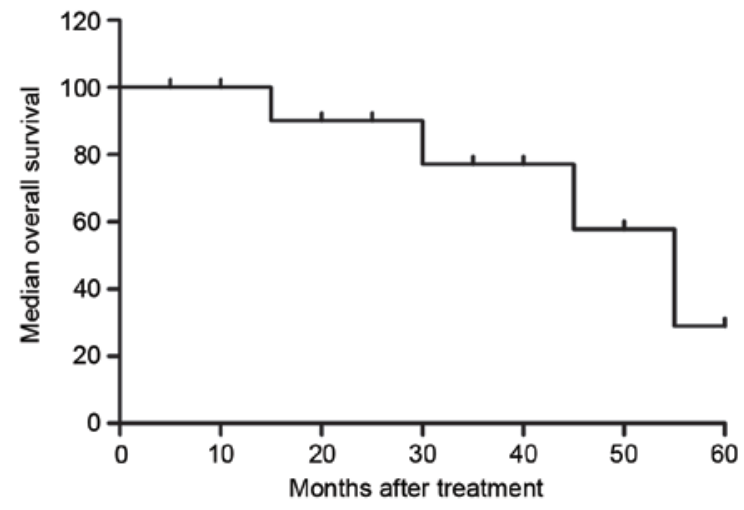

Figure 7. Evaluation the median overall survival of patients diagnosed by CECT-TNCA in early stage. Kaplan-Meier was used to estimate the survival rate during 60-months long-term observation. CECT-TNCA, contrast-enhanced computed tomography-targeting nanoparticles contrast agent.

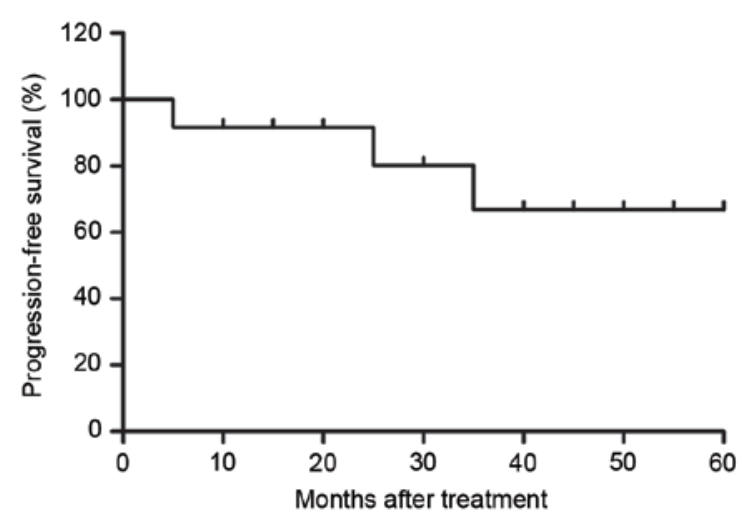

Figure 8. Analysis of the median progression-free survival of patients diagnosed by CECT-TNCA in early stage. Kaplan-Meier was used to estimate the survival rate during 60-months long-term observation. CECT-TNCA, contrast-enhanced computed tomography-targeting nanoparticles contrast agent.

and Kit have potent anti-tumor activity against a number of human tumors through binding with targets (44). Previous reports have suggested that target receptors of PDGFR- $\beta$, Ret, and $\mathrm{Kit}$ in gastric tumors are effective treatments (45-47). Although previous contrast media have not previously been compared to determine which media is optimal for the visualization and diagnosis of gastric cancer (48). Indeed, CECT using a different contrast agent enables non-destructive diagnosis of the biochemical and biomechanical properties of patients with various diseases (49). In addition, a previous study has evaluated dynamic CECT imaging in the differentiation of benign and malignant tumors observed by tumor vessel and permeability nodule perfusion (50). However, conventional contrast agents present lower efficacy for tumor analysis due to rapid diffusion outside the lungs, which prevents optimal imaging (51). Furthermore, previous reports have shown that iodinated contrast agents are less sensitive to changes in cells morphology $(52,53)$. Our design showed that liposome-encapsulated target nanoparticles contrast mediums are potential nanoparticles contrast agents that may improve the accuracy of diagnosing early-stage gastric tumors. Targeted binding of target nanoparticles contrast mediums with gastric tumor cells 
enhanced signal intensity in lesions in stomachs diagnosed by CECT-TNCA.

In conclusion, to the best of our knowledge, this is the first report of liposome-encapsulated targeted nanoparticles contrast mediums combined with CECT for the diagnosis of patients with suspected early stage gastric cancer. CECT-TNCA was administered orally to augment the signal intensity in the stomach, leading to a reliable and sensitive assessment of the tumor for clinical diagnosis in patients with gastric cancer.

\section{References}

1. Golpour S, Rafie N, Safavi SM and Miraghajani M: Dietary isoflavones and gastric cancer: A brief review of current studies. J Res Med Sci 20: 893-900, 2015.

2. Hossain MM and Chen Z: Comments on 'Application of an adaptive design to a randomized phase II selection trial in gastric cancer: A report of the study design' by Satoshi Morita and Junichi Sakamoto. Pharmaceutical Statistics. Pharm Stat 11: 267-268, 2012.

3. Khayatzadeh S, Feizi A, Saneei P and Esmaillzadeh A: Vitamin D intake, serum Vitamin D levels, and risk of gastric cancer: A systematic review and meta-analysis. J Res Med Sci 20: 790-796, 2015.

4. Pimenta-Melo AR, Monteiro-Soares M, Libânio D and Dinis-Ribeiro M: Missing rate for gastric cancer during upper gastrointestinal endoscopy: A systematic review and meta-analysis. Eur J Gastroenterol Hepatol 28: 1041-1049, 2016.

5. Veisani Y and Delpisheh A: Survival rate of gastric cancer in Iran; a systematic review and meta-analysis. Gastroenterol Hepatol Bed Bench 9: 78-86, 2016.

6. Nakajima T, Inokuchi K, Hattori T, Inoue K, Taguchi T, Kondou T, Abe O, Kikuchi K, Tanabe T and Ogawa N: Multi-institutional cooperative study of adjuvant immunochemotherapy in gastric cancer-five-year survival rate. Gan To Kagaku Ryoho 16 : 799-806, 1989, (Article in Japanese).

7. Jung SA, Park YM, Hong SW, Moon JH, Shin JS, Lee HR, Ha SH, Lee DH, Kim JH, Kim SM, et al: Cellular inhibitor of apoptosis protein 1 (cIAP1) stability contributes to YM155 resistance in human gastric cancer cells. J Biol Chem 290 9974-9985, 2015.

8. Izawa M, Mori T, Satoh T, Teramachi K and Sairenji T: Identification of an alternative form of caspase- 9 in human gastric cancer cell lines: A role of a caspase- 9 variant in apoptosis resistance. Apoptosis 4: 321-325, 1999.

9. Moody SE, Schinzel AC, Singh S, Izzo F, Strickland MR, Luo L, Thomas SR, Boehm JS, Kim SY, Wang ZC and Hahn WC: PRKACA mediates resistance to HER2-targeted therapy in breast cancer cells and restores anti-apoptotic signaling. Oncogene 34: 2061-2071, 2015

10. Shiota M, Yokomizo A and Naito S: Pro-survival and anti-apoptotic properties of androgen receptor signaling by oxidative stress promote treatment resistance in prostate cancer. Endocr Relat Cancer 19: R243-R253, 2012.

11. Yamato I, Sho M, Shimada K, Hotta K, Ueda Y, Yasuda S, Shigi N, Konishi N, Tsujikawa K and Nakajima Y: PCA-1/ALKBH3 contributes to pancreatic cancer by supporting apoptotic resistance and angiogenesis. Cancer Res 72: 4829-4839, 2012.

12. Kanat O, O'Neil B and Shahda S: Targeted therapy for advanced gastric cancer: A review of current status and future prospects. World J Gastrointest Oncol 7: 401-410, 2015.

13. Ebell MH, Culp MB and Radke TJ: A systematic review of symptoms for the diagnosis of ovarian cancer. Am J Prev Med 50: 384-394, 2016.

14. Mazzei MA, Guerrini S, Mazzei FG, Cioffi Squitieri N, Notaro D, de Donato G, Galzerano G, Sacco P, Setacci F, Volterrani L and Setacci C: Follow-up of endovascular aortic aneurysm repair: Preliminary validation of digital tomosynthesis and contrast enhanced ultrasound in detection of medium- to long-term complications. World J Radiol 8: 530-536, 2016.

15. Schalk SG, Demi L, Bouhouch N, Kuenen MPJ, Postema AW, de la Rosette JJMCH, Wijkstra H, Tjalkens TJ and Mischi M: Contrast-enhanced ultrasound angiogenesis imaging by mutual information analysis for prostate cancer localization. IEEE Trans Biomed Eng 64: 661-670, 2016.
16. Markic D, Krpina K, Ahel J, et al: Different presentations of renal cell cancer on ultrasound and computerized tomography. Urologia 81: 228-232, 2014

17. Shi H, Wang Z, Huang C, Gu X, Jia T, Zhang A, Wu Z, Zhu L, Luo X, Zhao X, et al: A functional CT contrast agent for in vivo imaging of tumor hypoxia. Small 12: 3995-4006, 2016.

18. Krylova NS, Demkina AE, Poteshkina NG and Khashieva FM Tissue Doppler imaging and ultrasonic methods for evaluating myocardial deformation in the diagnosis of hypertrophic cardiomyopathy. Kardiologiia 54: 79-84, 2014, (In Russian).

19. Chen CL, Hu GY, Mei Q, Qiu H, Long GX and Hu GQ: Epidermal growth factor receptor-targeted ultra-small superparamagnetic iron oxide particles for magnetic resonance molecular imaging of lung cancer cells in vitro. Chin Med J (Engl) 125: 2322-2328, 2012.

20. Shan L: Polyethylene glycol-coated and folic acid-conjugated superparamagnetic iron oxide nanoparticles. In: Molecular Imaging and Contrast Agent Database (MICAD), Bethesda (MD), 2004.

21. Nakamoto Y, Ishimori T, Sano K, Temma T, Ueda M, Saji H and Togashi K: Clinical efficacy of dual-phase scanning using Ga-DOTATOC-PET/CT in the detection of neuroendocrine tumours. Clin Radiol 71: 1069.e1-5, 2016.

22. Angelov KG, Vasileva MB, Grozdev KS, Sokolov MB and Todorov G: Clinical and pathological characteristics, and prognostic factors for gastric cancer survival in 155 patients in Bulgaria. Hepatogastroenterology 61: 2421-2424, 2014.

23. Dirani M, Nasreddine W, Abdulla F and Beydoun A: Seizure control and improvement of neurological dysfunction in Lafora disease with perampanel. Epilepsy Behav Case Rep 2: 164-166, 2014.

24. Kargahi N, Razavi SM, Deyhimi P and Homayouni S: Comparative evaluation of eosinophils in normal mucosa, dysplastic mucosa and oral squamous cell carcinoma with hematoxylin-eosin, Congo red, and EMR1 immunohistochemical staining techniques. Electron Physician 7: 1019-1026, 2015.

25. Soler M, Estevez MC, Villar-Vazquez R, Casal JI and Lechuga LM: Label-free nanoplasmonic sensing of tumor-associate autoantibodies for early diagnosis of colorectal cancer. Anal Chim Acta 930: 31-38, 2016.

26. Hopper AD and Campbell JA: Early diagnosis of oesophageal cancer improves outcomes. Practitioner 260: 23-28, 3, 2016.

27. Shah RB, Leandro G, Romerocaces G, Bentley J, Yoon J, Mendrinos S, Tadros Y, Tian W and Lash R: Improvement of Diagnostic Agreement among Pathologists in Resolving an 'Atypical Glands Suspicious for Cancer' diagnosis in prostate biopsies utilizing a novel 'Disease-Focused Diagnostic Review' quality improvement process. Hum Pathol 56: 155-162, 2016.

28. Haider MA, Yao X, Loblaw A and Finelli A: Multiparametric magnetic resonance imaging in the diagnosis of prostate cancer: A Systematic Review. Clin Oncol (R Coll Radiol) 28: 550-567, 2016.

29. Chen WG, Yang CM, Xu LH, Zhang N, Liu XY, Ma YG, Huo XL, Han YS, Tian DA and Zheng Y: Gene chip technology used in the detection of HPV infection in esophageal cancer of Kazakh Chinese in Xinjiang Province. J Huazhong Univ Sci Technol Med Sci 34: 343-347, 2014.

30. Li RZ, Shi JF, Zhou QZ, Wu RF, Li N, Wu LN, Zhou YQ, Wang Q, Liu ZH, Liu B and Qiao YL: Evaluation of gene chip technology for high risk type human papillomavirus in cervical cancer screening. Zhonghua Yi Xue Za Zhi 86: 307-311, 2006, (In Chinese).

31. Hampton T: Breast cancer gene chip study under way: Can new technology help predict treatment success? Jama 291: 2927-2930, 2004.

32. Watson RW: Gene-chip technology and prostate cancer: The identification of new genes regulating tumour progression. BJU Int 91: 307, 2003

33. Kooiman J, Sijpkens YW, de Vries JP, Brulez HF, Hamming JF, van der Molen AJ, Aarts NJ, Cannegieter SC, Putter H, Swarts R, et al: A randomized comparison of 1-h sodium bicarbonate hydration versus standard peri-procedural saline hydration in patients with chronic kidney disease undergoing intravenous contrast-enhanced computerized tomography. Nephrol Dial Transplant 29: 1029-1036, 2014.

34. Mir MA, Bali BS, Mir RA and Wani H: Assessment of the severity of acute pancreatitis by contrast-enhanced computerized tomography in 350 patients. Ulus Travma Acil Cerrahi Derg 19: 103-108, 2013. 
35. Kakimoto N, Chindasombatjaroen J, Tomita S, Shimamoto $H$ Uchiyama Y, Hasegawa Y, Kishino M, Murakami S and Furukawa S: Contrast-enhanced multidetector computerized tomography for odontogenic cysts and cystic-appearing tumors of the jaws: Is it useful? Oral Surg Oral Med Oral Pathol Oral Radiol 115: 104-113, 2013.

36. Freling N, Roele E, Schaefer-Prokop C and Fokkens W: Prediction of deep neck abscesses by contrast-enhanced computerized tomography in 76 clinically suspect consecutive patients. Laryngoscope 119: 1745-1752, 2009.

37. Konduru N, Keller J, Ma-Hock L, Gröters S, Landsiedel R, Donaghey TC, Brain JD, Wohlleben W and Molina RM: Biokinetics and effects of barium sulfate nanoparticles. Part Fibre Toxicol 11: 55, 2014.

38. Liss P, Hansell P, Fasching A and Palm F: Iodinated contrast media inhibit oxygen consumption in freshly isolated proximal tubular cells from elderly humans and diabetic rats: Influence of nitric oxide. Ups J Med Sci 121: 12-16, 2016.

39. Mishra SK, Kumar BS, Khushu S, Tripathi RP and Gangenahalli G: Increased transverse relaxivity in ultrasmall superparamagnetic iron oxide nanoparticles used as MRI contrast agent for biomedical imaging. Contrast Media Mol Imaging 11: 350-361, 2016.

40. Kitoh Y: 5. Inspection of hepatocellular carcinoma 3-Contrast for the diagnosis of hepatocellular carcinoma: Techniques of image contrast and the choice of MR contrast agent. Nihon Hoshasen Gijutsu Gakkai Zasshi 72: 441-451, 2016, (In Japanese).

41. Zhang J, Liu J, Wang LM, Li ZY and Yuan Z: Retroreflective-type Janus microspheres as a novel contrast agent for enhanced optical coherence tomography. J Biophotonics 10: 878-886, 2017.

42. Rini BI and Atkins MB: Resistance to targeted therapy in renal-cell carcinoma. Lancet Oncol 10: 992-1000, 2009.

43. Eichelberg C, Junker K, Ljungberg B and Moch H: Diagnostic and prognostic molecular markers for renal cell carcinoma: A critical appraisal of the current state of research and clinical applicability. Eur Urol 55: 851-863, 2009.

44. Hutson TE: Targeted therapies for the treatment of metastatic renal cell carcinoma: Clinical evidence. Oncologist 16 (Suppl 2): S14-S22, 2011

45. Pinto RP, Lima FK, Kulkzynski JM and Moreira LF: Expression of P16 and PDGFR-beta in gastric adenocarcinoma. Rev Col Bras Cir 36: 199-203, 2009, (In English, Portuguese).
46. Zhang F, Tang JM, Wang L, Wu PP and Zhang M: Immunohistochemical detection of RET proto-oncogene product in tumoral and nontumoral mucosae of gastric cancer. Anal Quant Cytopathol Histopathol 36: 128-136, 2014.

47. Borges Bdo N, Santos Eda S, Bastos CE, Pinto LC, Anselmo NP, Quaresma JA, Calcagno DQ, Burbano RM and Harada ML: Promoter polymorphisms and methylation of E-cadherin (CDH1) and KIT in gastric cancer patients from northern Brazil. Anticancer Res 30: 2225-2233, 2010.

48. Kingston MJ, Perriman DM, Neeman T, Smith PN and Webb AL: Contrast agent comparison for three-dimensional micro-CT angiography: A cadaveric study. Contrast Media Mol Imaging 11: 319-324, 2016.

49. Lakin BA, Patel H, Holland C, Freedman JD, Shelofsky JS, Snyder BD, Stok KS and Grinstaff MW: Contrast-enhanced CT using a cationic contrast agent enables non-destructive assessment of the biochemical and biomechanical properties of mouse tibial plateau cartilage. J Orthop Res 34: 1130-1138, 2016.

50. Sudarski S, Henzler T and Schoenberg SO: Post-therapeutic positron emission tomography/computed tomography for early detection of non-small cell lung cancer recurrence. Transl Lung Cancer Res 2: 295-303, 2013.

51. Hagberg GE, Mamedov I, Power A, Beyerlein M, Merkle H, Kiselev VG, Dhingra K, Kubìček V, Angelovski $G$ and Logothetis NK: Diffusion properties of conventional and calcium-sensitive MRI contrast agents in the rat cerebral cortex. Contrast Media Mol Imaging 9: 71-82, 2014.

52. Turetschek K, Preda A, Novikov V, Brasch RC, Weinmann HJ, Wunderbaldinger $\mathrm{P}$ and Roberts TP: Tumor microvascular changes in antiangiogenic treatment: Assessment by magnetic resonance contrast media of different molecular weights. J Magn Reson Imaging 20: 138-144, 2004.

53. Samei E, Saunders RS, Badea CT, Ghaghada KB, Hedlund LW, Qi Y, Yuan H, Bentley RC and Mukundan S Jr: Micro-CT imaging of breast tumors in rodents using a liposomal, nanoparticle contrast agent. Int J Nanomedicine 4: 277-282, 2009.

(i) $\Theta$ This work is licensed under a Creative Commons Attribution-NonCommercial-NoDerivatives 4.0 International (CC BY-NC-ND 4.0) License. 